\title{
¿Refleja Formicidae el impacto de la degradación de los bosques ribereños en la Amazonía Oriental?
}

\author{
Stefania Pinzón Triana ${ }^{1}$, Guillaume Xavier Rousseau ${ }^{1 *}$, Jhonatan Andrés Muñoz Gutiérrez², \\ Alexandra Rocha da Piedade ${ }^{3} \&$ Heder Braun $^{1}$ \\ 1. Programa de Pós-Graduação em Agroecologia, Universidade Estadual do Maranhão, São Luís, MA, Brasil; \\ stefaniapt@gmail.com,guilirous@yahoo.ca, hederbraun@gmail.com \\ 2. Politécnico Colombiano Jaime Isaza Cadavid, Medellín, Antioquia, Colombia; energiaselvatica@gmail.com \\ 3. Programa de Pós-Graduação BIONORTE, Universidade Federal do Maranhão, São Luis, Maranhão, Brasil; \\ alexandrarochapyer@gmail.com \\ * Correspondencia
}

Recibido 27-IX-2018. Corregido 09-I-2019. Aceptado 11-VII-2019.

\begin{abstract}
Does Formicidae reflect the impact of riparian forest degradation in the Eastern Amazon? The increasing anthropogenic pressure on Eastern Amazon makes imperative the diagnosis of forest degradation, particularly the effect on key communities within the riparian ecosystems, one of the last remaining Amazonian forests in Maranhão State. The Formicidae family is abundant in these ecosystems, plays a fundamental role in soil functions and is also considered a good indicator of land use changes. Therefore, this study aimed to determine the impact of riparian forest successional stages on the Formicidae richness, frequency, and composition. Sampling was performed during both dry and wet seasons using the TSBF method. Collected organisms were identified as morphospecies. Also, species-accumulation curves were created. Linear mixed models were used to evaluate the effects of seasonal, local, and successional stage on ant richness and frequency. Polynomial regression models were applied to investigate the relationship between ant richness and frequency with canopy cover and vegetation height. Lastly, the species composition was represented by the Jaccard similarity index. In total, we observed 1940 individuals grouped into 86 morphospecies. We obtained more than $80 \%$ of the probable species richness. Ant frequency and richness increased significantly, following the successional stage, with low values in the open areas, especially during the dry season. Canopy cover and vegetation height seemed to affect partially both Formicidae richness and frequency during the dry season. Intermediate and advanced successional areas presented similar species composition (with 50-shared species), followed by the areas of early succession (43) and anthropic use (34). We concluded that the elimination of riparian forests produces a considerable effect on the richness and frequency of the Formicidae family, mainly affecting them in open areas during the dry season, thus resulting in losses of $41 \%$ in richness and $56 \%$ in frequency in forest areas when they were transformed into agricultural systems. Nevertheless, succession restores forest structure and functions, thus favoring re-colonization of ant species. Formicidae reflects forest degradation and is a key group in monitoring programs for the conservation/restoration of local riparian forests.
\end{abstract}

Key words: Amazon riparian forest; succession; dry season; canopy cover; height vegetation; ants.

Pinzón Triana, S., Rousseau, G. X., Muñoz Gutiérrez, J. A., Rocha da Piedade, A., \& Braun, H. (2019). ¿Refleja Formicidae el impacto de la degradación de los bosques ribereños en la Amazonía Oriental?. Revista de Biología Tropical, 67(4), 850-860.

A pesar del compromiso asumido en la COP-21 para frenar la tala de bosques (BrasiliNDC, 2015), la tasa de deforestación en la Amazonia legal aumentó un $13.7 \%$ en el último año (2017-2018) (INPE, 2019), lo cual convirtió a Brasil en el país con mayor pérdida de cobertura vegetal con 1.33 Mha (Global Forest Watch, 2019). Se estima que tan solo, en el estado de Maranhão, $25211 \mathrm{~km}^{2}$ de la Amazonia legal fueron eliminados desde 1990 
(INPE, 2019). Ante este panorama, los bosques ribereños pueden considerarse uno de los ecosistemas más amenazados en la actualidad (Capon et al., 2013), ya que comúnmente constituyen las únicas zonas forestales remanentes en regiones antropizadas y sufren una fuerte presión por la continua ocupación debido a la facilidad de acceso al recurso hídrico (Nunes et al., 2014). Pese a existir leyes para su protección (Presidencia de la República de Brasil, 2012) y ser consideradas leyes muy blandas (Brancalion et al., 2016), estas no prevalecen ante la necesidad de subsistencia de las comunidades que dependen de estos ecosistemas como fuente de recursos para satisfacer sus necesidades fundamentales.

Como focos de diversidad (Rodrigues, Brancalion, \& Isernhagen, 2009) y otros servicios ecosistémicos (Lima \& Zakia, 2004; Ballinger \& Lake, 2006), estos ambientes de transición albergan comunidades consideradas de suma importancia para el funcionamiento general de los ecosistemas; por ejemplo, la macrofauna edáfica. Asimismo, algunos grupos de macroinvertebrados del suelo cumplen un papel ecológico fundamental y son considerados "ingenieros de los ecosistemas" (Jones, Lawton, \& Shachak, 1994). Según Bardgett et al. (2001), la presencia y actividad de estos organismos (con tamaño superior a $2 \mathrm{~mm}$ ) influyen directa e indirectamente en las rutas hidrológicas, la descomposición de la materia orgánica y su transferencia del suelo al agua. Por su parte, las hormigas (Hymenoptera: Formicidae), por medio de la construcción de corredores, cámaras y nidos, aumentan la porosidad del suelo, la captura y el drenaje del agua - tanto en la superficie como en sus horizontes internos - y favorecen la mineralización de nutrientes (Folgarait, 1998; Bardgett et al., 2001). También contribuyen en la dispersión, germinación de semillas y viabilidad de plántulas (Dáttilo, Marques, Falcão, \& Moreira, 2009; Leal, Leal, \& Andersen, 2015; Tanaka, Ogata, Mukai, Yamawo, \& Tokuda, 2015), así como en el control de poblaciones de artrópodos y la polinización (Hölldobler \& Wilson,
1994; Del-Claro, Rodriguez-Morales, Calixto, Martins, \& Torezan-Silingardi, 2019).

No obstante, la presencia y actividad de formícidos es afectada por la mudanza en el componente forestal (Vasconcelos, 1999; Neves, Queiroz-Dantas, Rocha, \& Delabie, 2013; Leal, Silva, Sousa-Souto, \& Neves, 2016). Con la reducción de la heterogeneidad del ecosistema, se disminuye la complejidad de los microhábitats y la diversidad de alimentos, limitando así el forrajeo y nidificación de estos organismos (Vasconcelos \& Vilhena, 2006). El forrajeo y la nidificación son actividades íntimamente ligadas con la hojarasca que cubre el suelo (García-Martínez, Escobar-Sarria, LópezBarrera. Castaño-Meneses, \& Valenzuela-González, 2015, 2017) y la presencia de plantas con las que interactúan (Lopes et al., 2012). De esta forma, tras un impacto antropogénico como la tala de la vegetación, la compactación del suelo aumenta e influye negativamente en el establecimiento de hormigas epígeas en bosques ribereños y en la dinámica de desplazamiento de especies a escala del paisaje (García-Martínez et al., 2015, 2017).

Debido a que la familia Formicidae cumple un papel fundamental en estos ambientes (Bardgett et al., 2001; García-Martínez et al., 2017), refleja los cambios a nivel estructural y funcional del suelo (García-Martínez et al., 2015), es abundante (Pinzón et al., 2015) y son pocos los estudios realizados en bosques ribereños, el presente trabajo tiene como objetivos evidenciar el aporte referencial de Formicidae en estas zonas y establecer diferencias en su riqueza, frecuencia y composición a través del gradiente de sucesión forestal. Las hipótesis consideradas en este estudio fueron que (1) existe una relación positiva entre el gradiente de sucesión forestal y la riqueza y frecuencia de Formicidae; (2) las áreas de sucesión comparten mayor número de especies y se distancian de áreas de uso antrópico; (3) la riqueza y frecuencia de este grupo es afectada por la cobertura de dosel, el altura de los árboles y el periodo de recolecta. 


\section{MATERIALES Y MÉTODOS}

Área de estudio: El trabajo se realizó

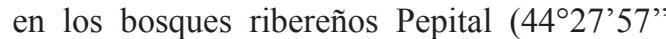

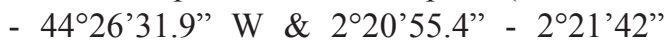

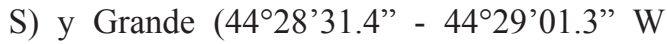
\& 2²0'49.5” - 2²0'52.7" S), los cuales se encuentran severamente degradados por actividades agro-extractivistas (Zelarayán et al., 2015). Las dos cuencas están situadas en el municipio de Alcântara al norte del estado de Maranhão, Brasil, y comprenden vegetación ombrófila abierta del bioma Amazonia Oriental (IBGE, 2004).

Diseño experimental: Se categorizaron visualmente cuatro niveles de sucesión forestal basados en la estimación de la altura media de la vegetación y la abertura del dosel (Celentano et al., 2017). También, se establecieron tres bloques de muestreo por bosque, cada uno con cuatro parcelas de $50 \times 20 \mathrm{~m}$ - una para cada nivel de sucesión forestal - sumando un total de 24. Estas últimas correspondían a la sucesión 1 (áreas abiertas o de uso antrópico, con cultivos o pastizales y cobertura del dosel menor a 19\%); sucesión 2 (matorral menor a 7 $\mathrm{m}$, cobertura del dosel entre 20 y $69 \%$ ); sucesión 3 (bosque secundario entre 7 y $15 \mathrm{~m}$ con dosel entre 70 y $89 \%$ ); y la sucesión 4 (bosque secundario mayor a $15 \mathrm{~m}$ con más del $90 \%$ del dosel). Las parcelas fueron establecidas distantes del lecho del río (mínimo $7 \mathrm{~m}$ ) para evitar posibles inundaciones (Celentano et al., 2017).

Como parte de un programa de muestreo de macrofauna edáfica, la recolecta se llevó a cabo durante los períodos seco de noviembre 2012 (precipitación media mensual de 5.25 $\mathrm{mm}$ ) y lluvioso de junio 2013 (precipitación media mensual de $94.25 \mathrm{~mm}$ ) (datos proporcionados por el LabMet/UEMA). Para al muestreo, se empleó una versión modificada del método Tropical Soil Biology and Fertility - TSBF (Anderson \& Ingram, 1993) que consistió en la extracción de 5 monolitos de $25 \mathrm{~cm}^{2}$ y $10 \mathrm{~cm}$ de profundidad retirados en zigzag a 2 $\mathrm{m}$ de la línea media de la parcela (más detalles en Pinzón et al., 2015).
Los formícidos, tanto del suelo como de la hojarasca, fueron separados de la macrofauna, contados y determinados hasta el nivel de especies y morfoespecies. Los ejemplares fueron posteriormente identificados y convalidados por el especialista Jacques Delabie en el Laboratorio de Mirmecología en el Centro de Investigación de Cacao-CEPLAC en llhéus, Bahia, Brasil. Actualmente, el material forma parte de la Colección de Invertebrados del Suelo de Maranhão-CISMA, inscrita al Laboratorio de Artrópodos y Microbiología del Suelo-LAMS de la Universidad Estadual de MaranhãoUEMA en São Luis, Maranhão, Brasil.

Análisis estadísticos: Fueron construidas curvas de acumulación de especies (o de recolector) con 100 aleatorizaciones que evalúan la eficiencia del método usado para representar la subcomunidad de hormigas en los bosques ribereños, con la adición de nuevas especies en función del esfuerzo amostral. Las curvas y sus desviaciones estándar se calcularon con el paquete estadístico EstimateS versión 9.1.0 (Colwell, 2013). Por otro lado, para estimar la riqueza de especies fueron utilizados Chao 2 y Jackknife 1.

Los datos de abundancia fueron analizados utilizando la frecuencia reportada en los registros de las especies en cada área muestreada y no por el número de individuos. Esta medida es considerada el parámetro más adecuado para insectos sociales debido a la divergencia entre el número de individuos por colonia (Longino, Coddington, \& Colwell, 2002). Asimismo, debido a la corta distancia, los cinco monolitos fueron considerados como una única muestra por parcela para evitar el reconteo de individuos de la misma colonia y sobreestimar la frecuencia relativa por nivel de sucesión forestal.

Tras observar la homogeneidad de las varianzas de los residuos, fueron realizados Modelos Lineales Mixtos (LMM, siglas en inglés) para establecer el efecto de la interacción entre la sucesión, la temporada de recolecta y el bosque ribereño en la riqueza y frecuencia de Formicidae. Al confirmar que no hubo efecto significativo por parte del bosque 
ribereño (Pepital o Grande), el modelo fue establecido de la siguiente forma: los cuatro niveles de sucesión (1-4) y el período de recolecta (seco y lluvioso) fueron considerados como factores fijos, mientras tanto, el bloque (tres por bosque) como factor aleatorio. Este modelo fue escogido debido a que obtuvo el valor más bajo en el criterio de información Akaiko (Di Rienzo, Macchiavelli, \& Casanoves, 2009).

Posteriormente, los promedios fueron comparados mediante la prueba de Tukey ( $\mathrm{p} \leq$ $0.05)$. El análisis fue realizado con el paquete multcomp (Hothorn et al., 2016) de la plataforma R (R Development Core Team, 2009). También, fueron realizadas regresiones polinómicas para determinar la relación entre las variables riqueza y frecuencia de hormigas con la cobertura del dosel y la altura de la vegetación ya que, de acuerdo con Celentano et al. (2017), estas variables estructurales fueron las que reflejaron el estado de degradación forestal local. Estos análisis se hicieron con ayuda del software Minitab 18 (Minitab, 2018).

Finalmente, la semejanza en la composición de especies fue representada a través del índice de similitud de Jaccard, el más indicado en matrices de datos de presencia - ausencia (Legendre, Borcard, \& Peres-Neto, 2005) y usado para observar el grado de semejanza entre los niveles de sucesión y las parcelas amostradas. Estos análisis fueron realizados a través del software Primer v7 (Clarke \& Gorley, 2015) para las cuatro sucesiones y con el paquete vegan (Oksanen et al., 2018) del software R (R Development Core Team, 2009) para las 24 parcelas.

\section{RESULTADOS}

Se muestreó un total de 1940 individuos separados en 86 especies y morfoespecies, 15 tribus y 8 subfamilias de las 14 conocidas para el Neotrópico (Apéndice 1). De los 663 registros totales, las especies más frecuentes fueron Pheidole radoszkowskii (60 registros), Nylanderia sp.1 (43), Hypoponera sp.1 (34), Hypoponera sp.3 (32) y Brachymyrmex sp.1 (30). Por otro lado, los géneros Pseudomyrmex y Camponotus fueron los más diversos con 9 y 8 morfoespecies respectivamente. Estos últimos representan el $21 \%$ del número de especies recolectadas. Asimismo, Hypoponera, Pheidole y Solenopsis, con 5 morfoespecies cada uno, sumados corresponden al $17 \%$ del número total de especies encontradas. Por otra parte, 15 morfoespecies fueron registradas una única vez (singletons) y equivalían al $16 \%$ de la riqueza total.

Las curvas de acumulación de especies mostraron una estabilización con ambos estimadores de riqueza Jackknife 1 y Chao 2 (Apéndice 2). Por lo tanto, se constató que, con el método de recolecta, se alcanzó el 84.1 y $92.6 \%$ (Jackknife 1 y Chao 2, respectivamente) de los valores estimados para el número de especies probables en la subcomunidad local de hormigas. También, se determinó que el modelo estadístico usado para los LMM se ajustó de manera adecuada (a través del test chi cuadrado - $\chi^{2}$ Wald Tipo II) al considerar la interacción del nivel de sucesión por período para los datos de riqueza $\left(\chi^{2}=9.08\right.$, g.l. $=3, \mathrm{P}$ $=0.028)$ y frecuencia $\left(\chi^{2}=12.62\right.$, g.l. $=3, P=$ $0.005)$ de Formicidae.

De acuerdo con los resultados, las medias de riqueza y frecuencia fueron significativamente más altas en el nivel de sucesión más avanzado (4) durante el periodo seco (Media Riqueza $=15.17, \mathrm{ES}=1.24$; Media Frecuencia $=23.69, \mathrm{ES}=2.12$ ), las cuales se diferenciaron significativamente de los valores presentados en el nivel de sucesión temprana (1) en el mismo periodo (Media Riqueza $=5.31, \mathrm{ES}=$ 1.21; Media Frecuencia $=7.32$, ES $=2.09$ ), como se observa en la Fig. 1A y la Fig. 1B.

Asimismo, los resultados de las regresiones polinómicas (Apéndice 3) mostraron que el 62 $\%$ de la variabilidad de la riqueza de hormigas fue explicada por el modelo ajustado en relación con el porcentaje de cobertura del dosel $\left(\mathrm{r}^{2}\right.$ $=0.62, \mathrm{~F}=10.70, \mathrm{P}<0.0001)$ durante el período seco, un $58 \%$ la frecuencia de hormigas $\left(\mathrm{r}^{2}\right.$ $=0.58, \mathrm{~F}=9.05, \mathrm{p}=0.001)$. De igual manera, la altura de la vegetación tuvo un efecto moderado en relación con la riqueza y la fre $\neg$ cuencia de especies de hormigas $\left(\mathrm{r}^{2}=0.52, \mathrm{~F}=7.31\right.$, 

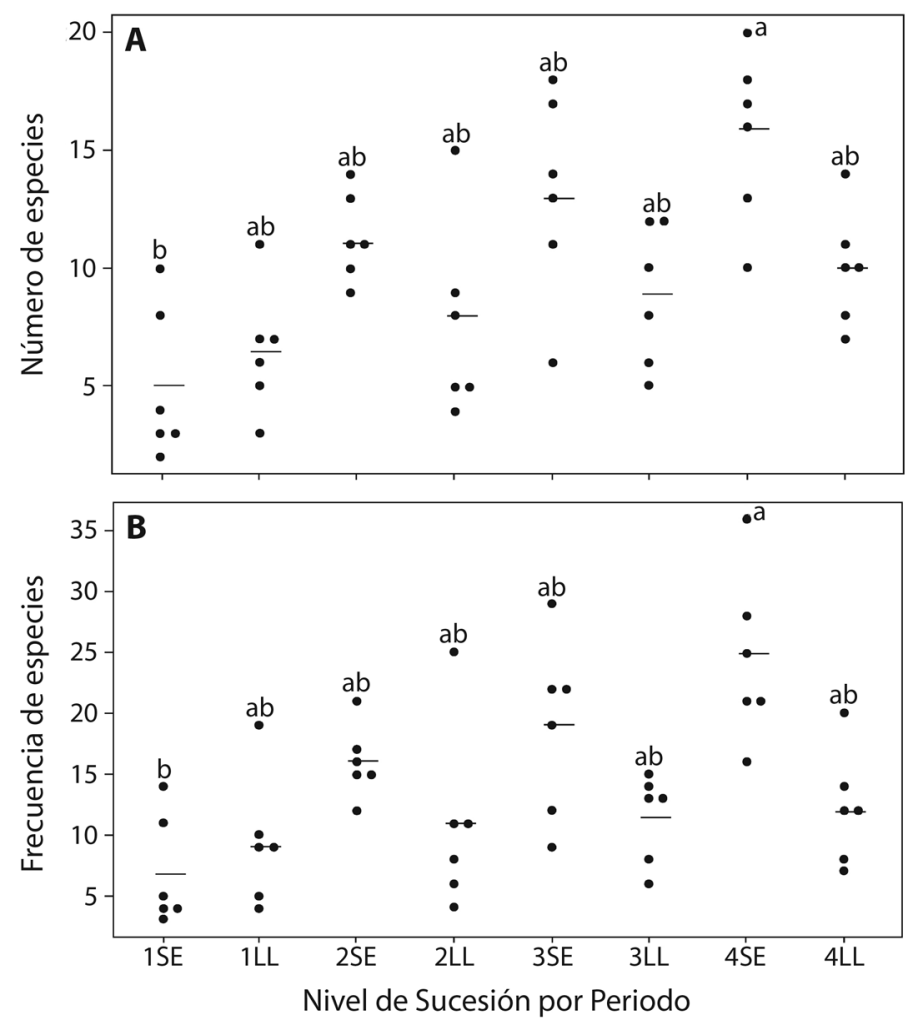

Fig. 1. A) Variabilidad de la riqueza y B) frecuencia de Formicidae en los niveles de sucesión vegetal (1-4) y el período de recolecta (LL: periodo lluvioso, SE: periodo seco) en los bosques ribereños Pepital y Grande, Alcântara, Maranhão. Los puntos con letras diferentes en el mismo grupo fueron estadísticamente distintos al $5 \%$ de probabilidad de acuerdo con la prueba de Tukey.

Fig. 1. A) Variability of Formicidae richness and B) frequency in the vegetation sucessional levels (1-4) and the sampling season (LL: rainy season, SE: dry season) in Pepital and Grande riparian forests, Alcântara, Maranhão. The points with different letters in the same group were statistically different at $5 \%$ probability, according to the Tukey test.

$\mathrm{P}=0.002 ; \mathrm{r}^{2}=0.52, \mathrm{~F}=7.13, \mathrm{P}=0.002$ ) en el mismo período. Cuando se consideraron las dos épocas juntas y solamente el período lluvioso, el ajuste de los modelos fue bajo.

Por otra parte, según el análisis de similitud de Jaccard para la composición de especies de Formicidae en los cuatro niveles sucesionales (Tabla 1) se observó que: la sucesión 1 fue la más disímil con solo $40 \%$ de especies compartidas con las demás (correspondiente a 34 spp.); la sucesión 2 con $50 \%$ de semejanza (43 spp.); las sucesiones 3 y 4 compartieron un $58 \%$ del total (50 spp.). El Coeficiente de Correlación Cofenética (CCC) para este agrupamiento mostró un grado de fidelidad de $89 \%$ $(\mathrm{CCC}=0.89)$.
TABLA 1

Valores de riqueza de especies (en la diagonal), índice de similitud de Jaccard (debajo de la diagonal) y número de especies compartidas de Formicidae (encima de la diagonal) en los diferentes niveles de sucesión (CCC: 0.89)

\section{TABLE 1}

Values of species richness (diagonal), Jaccard similarity index (below the diagonal) and number of shared species of Formicidae (above the diagonal) in different degradation levels (CCC: 0.89)

\begin{tabular}{ccccc} 
Nivel de sucesión & 1 & 2 & 3 & 4 \\
1 & 37 & 15 & 13 & 16 \\
2 & 0.413 & 52 & 24 & 29 \\
3 & 0.359 & 0.452 & 54 & 31 \\
4 & 0.429 & 0.554 & 0.581 & 63 \\
\hline
\end{tabular}


Por otro lado, el clúster de especies compartidas entre parcelas mostró una agrupación de cinco áreas (Fig. 2). La mayor parte de las sucesiones 3 y 4 en las dos cuencas se encontraban en un grupo que compartió el $28 \%$ de semejanza en la composición de especies. Con dos representantes por nivel, las sucesiones temprana, intermediaria y avanzada (2-4) se agruparon y presentaron una similitud cercana al $26 \%$. Además, para los niveles de sucesión 1 y 2, se observan dos aglomerados: uno donde tres de las parcelas de uso antrópico (1) se agruparon con dos parcelas de la sucesión temprana (2) de las dos cuencas, representando un $25 \%$ de semejanza y otro de dos parcelas de sucesión 1 y 2 con semejanza del $20 \%$. Por último, el grupo formado por las parcelas de sucesión 1 y 3 del bosque Pepital (con un 14 \% de especies compartidas) fue el más disímil ya que se separó del resto.

\section{DISCUSIÓN}

De acuerdo con los resultados, la abundancia total de hormigas fue menor que lo observado por Oliveira, Silva, \& Santana (2014) en bosques ribereños y de galerías del Cerrado brasilero, quienes registraron más de $10 \mathrm{mil}$ individuos con los métodos de recolecta tradicionales para este grupo (trampas de caída Pitfall y cebos con sardina). No obstante, la riqueza registrada en dicho estudio fue de solamente 47 especies; en este estudio se identificaron $86 \mathrm{spp}$. Por otra parte, usando TSBF, se obtuvieron valores cercanos a los de Rousseau, Silva, Celentano, \& Carvalho (2014)

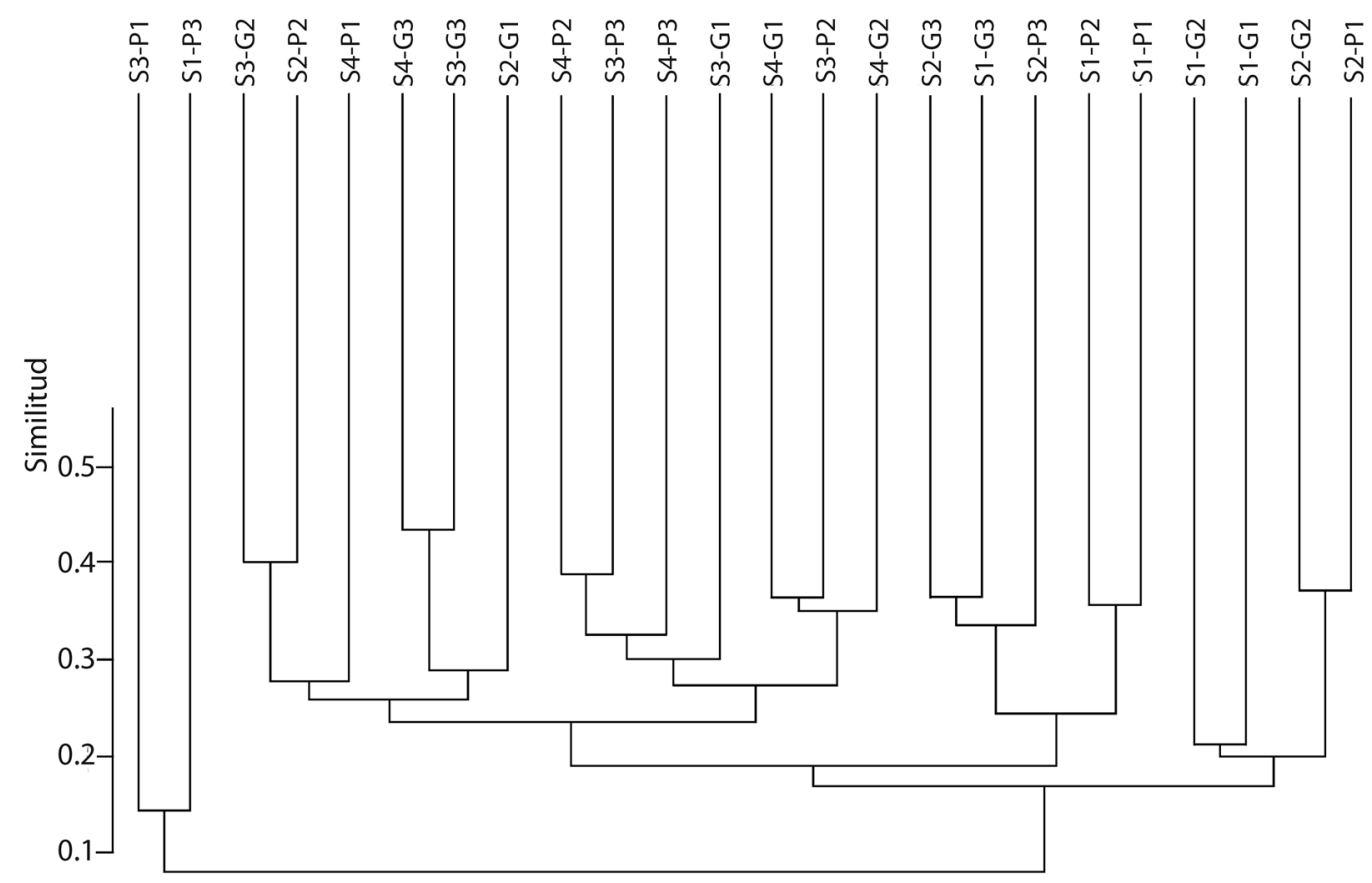

Fig. 2. Agrupamiento de las parcelas en función de la composición de especies de hormigas, basado en el índice de similitud de Jaccard (CCC: 0.79). Sucesión 1-S1: áreas abiertas, cultivos o pastizales; sucesión 2-S2: matorral menor a $7 \mathrm{~m}$ de altura; sucesión 3-S3: bosque secundario entre 7 m y 15 m; sucesión 4-S4: bosque secundario mayor a 15 m. P1, P2, P3: repeticiones del bosque ribereño Pepital; G1, G2, G3: repeticiones del bosque ribereño Grande.

Fig. 2. Cluster of plots according to the composition of ant species, based on the Jaccard similarity index (CCC: 0.79). Succession 1-S1: open areas, crops or pastures; succession 2-S2: shrub below $7 \mathrm{~m}$ height; succession 3-S3: secondary forest between $7 \mathrm{~m}$ and $15 \mathrm{~m}$; succession 4-S4: secondary forest over $15 \mathrm{~m}$. P1, P2, P3: repetitions of Pepital riparian forest; G1, G2, G3: repetitions of Grande riparian forest. 
en pastajes, bosques primarios y de sucesión secundaria en la Amazonia Oriental con 2186 individuos registrados.

Las subfamilias Myrmicinae y Formicinae fueron las más frecuentes, caracterizadas por la diversidad de hábitos alimenticios y preferencia particular de hábitats (Baccaro et al., 2015). En esta línea, la diversidad de especies de los géneros Pseudomyrmex y Camponotus puede deberse a la amplia distribución de las especies de estos grupos, su alimentación generalista y la capacidad de habitar ambientes con distintos grados de antropización, comúnmente presentes en la vegetación y la hojarasca (Baccaro et al., 2015). Sin embargo, de las especies recolectadas en el presente estudio, 44 (equivalente al $51 \%$ ), tienen hábito hipogéico o críptico de acuerdo con Schmidt \& Solar (2010). Esto puede deberse al método de recolecta utilizado.

Aunque el TSBF considera el procesamiento de un volumen mayor de suelo en relación con la hojarasca, lo cual demanda un mayor tiempo y esfuerzo, las especies no edáficas (arborícolas, nómades y de la hojarasca) tuvieron una buena representación (49\%) en este estudio, de modo que la relación costobeneficio de su uso fue favorable. Por otro lado, a pesar que el TSBF no es indicado como método de muestreo específico para hormigas, las curvas de acumulación de especies indicaron una estimación promedio del $88 \%$ de las especies probables localmente. Estos estimadores utilizados son indicadores de riqueza eficientes en otros estudios de diversidad de Formicidae (Lutinski, Lutinski, Iop, \& Garcia, 2013; Vasconcelos et al., 2014). Asimismo, de acuerdo con Melo (2004), son los menos influenciados por el tamaño amostral de las especies.

No obstante, la estabilización de estas curvas puede presentarse sin que muchas especies hayan sido registradas debido al turnover (entrada y salida) de especies en gradientes ambientales, la presencia natural de especies muy raras (Whittaker, 1972) y la estabilidad espacio-temporal de las especies (Longino et al., 2002). De esta forma, el TSBF tiene un gran potencial para la recolecta de estos organismos y puede representar un complemento eficiente para los métodos tradicionalmente utilizados. Métodos como las trampas de caída o atrayentes no permiten recolectar formícidos hipogéicos (Schmidt \& Solar, 2010), hormigas de hábito subterráneo que suscitan un alto interés por considerarse la "frontera final" en estudios de diversidad de Formicidae (Wilkie, Mertl, \& Traniello, 2007). Sin embargo, es necesario realizar estudios comparando la eficiencia del TSBF respecto a otros métodos de muestreo.

Por otra parte, el efecto del período lluvioso se evidenció en la disminución tanto en la riqueza como en la frecuencia de hormigas (con pérdidas de 11 especies y 137 registros). Esto puede deberse a que en algunas parcelas de sucesión avanzada se registró un alto contenido de carbono y limo (producto de la vegetación) y, por ende, una mayor retención de agua en el suelo (Celentano et al., 2017). Esto pudo ocasionar un desplazamiento parcial de especies debido a la pérdida de sitios de nidificación, condición básica para el sustento de estos organismos en los ecosistemas (Vasconcelos \& Vilhena, 2006). Estos resultados son contrarios a Vasconcellos, Segat, Bonfim, Baretta, \& Cardoso (2013) donde se observó un aumento en la riqueza durante el período lluvioso en bosques ribereños de Mata Atlántica. No obstante, García-Martínez et al. (2015) registraron un aumento en la riqueza y la frecuencia de hormigas durante el periodo seco en bosques ribereños altamente intervenidos.

Otra razón que justifica la disminución de riqueza (con pérdidas que variaron de $24 \%$ a $41 \%$ ) y frecuencia (pérdidas de $40 \%$ a $56 \%$ ) de hormigas puede ser la transformación de bosques en sistemas agropecuarios. Esta variación dependió del grado de sucesión vegetal, revelando que cuánto más avanzada fuera la sucesión secundaria, mayor número y frecuencia de especies de hormigas se encontraban y fueron perdidas. Es importante tener en cuenta que la selección de las variables de cobertura de dosel y altura de la vegetación se hizo debido a que fueron indicadoras del estado de degradación/conservación de estos bosques como se muestra en Celentano et al., (2017). La 
riqueza de especies de árboles y arbustos (no presentada en este estudio) no fue superior a las variables abordadas por lo cual se descartó.

Por lo tanto, la pérdida natural e inducida de la cobertura del dosel parece responder en parte a esta disminución, ya que su efecto se hizo más notorio durante el período seco y en las áreas de uso antrópico. La sequía torna los ambientes menos heterogéneos, con menor diversidad estructural en áreas degradadas, lo que resulta en menos hábitats y alimento disponible y un aumento en la competitividad intra e inter específica (Oliveira et al., 2014). En ese sentido, la similitud en la riqueza y composición de especies de Formicidae entre las áreas de sucesión media y avanzada (3 y 4) podría deberse a la semejanza estructural de la vegetación.

Además, puede asumirse que los bosques ribereños estudiados todavía representan un continuum para muchas especies debido a que el grado de fragmentación y aislamiento no es tan elevado, facilitando así la colonización y turnover de las especies entre el gradiente sucesional. En el estudio de Vasconcelos, Vilhena, Magnusson, \& Albernaz (2006), se ha mostrado que en fragmentos Amazónicos distanciados hasta en $300 \mathrm{~m}$, ocurrió un turnover gracias al estado de conservación y tamaño de los fragmentos. De igual manera, el efecto de borde también fue registrado en bosques ribereños por Gollan, De Bruyn, Reid, Smith, \& Wilkie (2011), quienes afirman que no hubo diferencia estadística entre la riqueza de hormigas y el gradiente de sucesión vegetal.

Para concluir, este estudio mostró una aproximación satisfactoria de la riqueza de hormigas presentes en los bosques ribereños estudiados considerando el método de recolecta usado, cuyo costo-beneficio fue favorable. También, se determinó que hubo efecto del período seco en áreas de uso antrópico en la comunidad de hormigas, evidenciado en la disminución de la riqueza y frecuencia de estos organismos. Este detrimento en la riqueza y frecuencia se observó de manera más marcada en el periodo seco por la pérdida natural e inducida en la cobertura del dosel. Así entonces, el incremento de la diversidad de hormigas de acuerdo con la sucesión puede indicar una mejoría de las condiciones microclimáticas en cuanto a la oferta de alimento y hábitats que favorecen la diversidad de estos organismos.

Finalmente, este grupo reflejó el impacto a nivel poblacional que tiene la degradación de los bosques ribereños, ecosistemas claves para la manutención del suministro de bienes y servicios indispensables para las comunidades humanas. Por ello, deben considerarse prácticas agropecuarias en las tierras adyacentes a los bosques ribereños, como los sistemas agroforestales y silvopastoriles, que medien la necesidad de las personas de garantizar su subsistencia con un bajo impacto ambiental.

Declaración de ética: los autores declaran que todos están de acuerdo con esta publicación y que han hecho aportes que justifican su autoría; que no hay conflicto de interés de ningún tipo; y que han cumplido con todos los requisitos y procedimientos éticos y legales pertinentes. Todas las fuentes de financiamiento se detallan plena y claramente en la sección de agradecimientos. El respectivo documento legal firmado se encuentra en los archivos de la revista.

\section{AGRADECIMIENTOS}

Los autores agradecen a las agencias CAPES y FAPEMA por el apoyo financiero brindado. A Fabricio Baccaro, Jorge Souza, Joudellys Silva y Jacques Delabie quienes apoyaron el proceso de identificación y confirmación de especies. Al equipo completo de estudiantes e investigadores del Grupo de Investigación de Alcântara. Al posgrado en Agroecología y LabMet de la UEMA. A las comunidades de Pepital e Só Assim. A los revisores que contribuyeron en la mejora del presente manuscrito. 


\section{RESUMEN}

La creciente presión antropogénica sobre la Amazonia Oriental hace imperativo el diagnóstico de la degradación forestal $\mathrm{y}$, particularmente, su efecto sobre las comunidades clave dentro de los ecosistemas ribereños, unos de los últimos bosques amazónicos remanentes en el estado de Maranhão. La familia Formicidae juega un papel fundamental en el suelo, refleja los cambios en el uso de la tierra y es un grupo abundante en los bosques estudiados. Este trabajo tuvo como objetivo determinar el efecto del gradiente sucesional en la riqueza, frecuencia y composición de esta familia. El muestreo fue realizado durante los periodos seco y lluvioso con el método TSBF. Los organismos recolectados se identificaron a nivel de morfoespecies y se crearon curvas de acumulación de especies. Fueron utilizados modelos lineales mixtos para evaluar los efectos del periodo de recolecta local y estado de sucesión en la riqueza y la frecuencia de las hormigas. Por otro lado, se utilizaron regresiones polinómicas para investigar la relación entre la riqueza y la frecuencia de los formícidos según la cobertura del dosel y la altura de la vegetación. La composición de especies fue representada a través del índice de similitud de Jaccard. En total, 1940 individuos fueron separados en 86 morfoespecies. Se obtuvo más del $80 \%$ de la riqueza de especies probables. La frecuencia y riqueza de hormigas aumentó significativamente siguiendo la sucesión con valores bajos en las áreas abiertas durante la estación seca. Asimismo, durante esta estación, la cobertura del dosel y la altura de la vegetación tuvieron un efecto parcial en la riqueza y frecuencia de Formicidae. Por su parte, las áreas de sucesión intermedia y avanzada presentaron una composición similar con 50 especies compartidas, seguidas de las áreas de sucesión temprana con 43 y el uso antrópico con 34. Concluimos que la eliminación de los bosques ribereños tiene un efecto considerable sobre la riqueza y frecuencia de Formicidae, con valores mínimos en áreas degradadas durante la estación seca. Por otro lado, las áreas de bosques transformadas en sistemas agrícolas sufrieron pérdidas de 41 y $56 \%$ en la riqueza y frecuencia respectivamente. Con la sucesión, se restauran la estructura y las funciones del bosque favoreciendo la recolonización de las especies de hormigas. Finalmente, estos himenópteros son un grupo clave en los programas de monitoreo para la conservación/restauración de los bosques ribereños locales.

Palabras claves: bosque ribereño amazónico; sucesión; estación seca; cobertura de dosel; altura de la vegetación; hormigas.

\section{REFERENCIAS}

Anderson, J. M., \& Ingram, J. S. I. (1993). Field Procedures. En J. M. Anderson, \& J. S. I. Ingram (Eds.), Tropical Soil Biology and Fertility: a handbook of methods (pp. 44-46). Wallingford, England: CAB International.

Baccaro, F. B., Feitosa, R. M., Fernandez, F., Fernandes, I. O., Izzo, T. J., Souza, J. L. P., \& Solar, R. (2015). Guia para os gêneros de formigas do Brasil. Manaus, Brasil: Editora INPA.

Ballinger, A., \& Lake, P. S. (2006). Energy and nutrient fluxes from rivers and streams into terrestrial food webs. Marine and Freshwater Research, 57, 15-28.

Bardgett, R. D., Anderson, J. M., Behan-Pelletier, V., Brussaard, L., Coleman, D. C., ... Wall, D. H. (2001). The influence of soil biodiversity on hydrological pathways and the transfer of materials between terrestrial and aquatic ecosystems. Ecosystems, 4(5), 421-429.

Brancalion, P. H. S., Garcia, L. C., Loyola, R., Rodrigues, R. R., Pillar, V. D., \& Lewinsohn, T. M. (2016). A critical analysis of the Native Vegetation Protection Law of Brazil (2012): updates and ongoing initiatives. Natureza e Conservação, 14, 1-15.

Presidencia de la República de Brasil. (2012). Código florestal brasileiro (Lei $\mathrm{N}^{\circ} 12.651$ de 25 de Maio de 2012). Recuperado de http://www.planalto.gov.br/ ccivil_03/_ato2011-2014/2012/lei/112651.htm

BRASIL-iNDC. (2015). Pretendida contribuição nacionalmente determinada para consecução do objetivo da convenção-quadro das nações unidas sobre mudança do clima. Recuperado de http://www.itamaraty.gov. br/images/ed_desenvsust/BRASIL-iNDC-portugues. pdf

Capon, S. J., Chambers, L. E., Mac Nally, R., Naiman, R. J., Davies, P., Marshall, N., ... Williams, S. E. (2013). Riparian Ecosystems in the 21st Century: Hotspots for Climate Change Adaptation? Ecosystems, 16(3), 359-381.

Celentano, D., Rousseau, G. X., Engel, V. L., Zelarayán, M., Oliveira, E. C., Araujo, A. C. M., \& de Moura, E. G. (2017). Degradation of riparian forest affects soil properties and ecosystem services provision in Eastern Amazon of Brazil. Land Degradation and Development, 28, 482-493.

Clarke, K. R., \& Gorley, R. N. (2015). PRIMER v7: User Manual/Tutorial. Plymouth, England: PRIMER-E.

Colwell, R. K. (2013). EstimateS, version 9.1: statistical estimation of species richness and shared species from samples (software and user's guide). Recuperado de http://viceroy.eeb.uconn.edu/estimates

Del-Claro, K., Rodriguez-Morales, D., Calixto, E. S., Martins, A. S., \& Torezan-Silingardi, H. M. (2019). Ant pollination of Paepalanthus lundii (Eriocaulaceae) in Brazilian savanna. Annals of Botany, mcz021, 1-7. DOI: $10.1093 / \mathrm{aob} / \mathrm{mcz} 021$ 
Dáttilo, W., Marques, E. C., Falcão, J. C. F., \& Moreira, D. D. O. (2009). Interações mutualísticas entre formigas e plantas. EntomoBrasilis, 2(2), 32-36.

Di Rienzo, J. A., Macchiavelli, R., \& Casanoves, F. (2009). Modelos Mixtos InfoStat. Recuperado de academic. uprm.edu/rmacchia/agro6998/TutorialMMesp.pdf

Folgarait, P. J. (1998). Ant biodiversity and its relationship to ecosystem functioning: A review. Biodiversity and Conservation, 7(9), 1221-1244.

García-Martínez, M. A., Escobar-Sarria, F., López-Barrera, F., Castaño-Meneses, G., \& Valenzuela-González, J. E. (2015). Value of riparian vegetation remnants for leaf-litter ants (Hymenoptera: Formicidae) in a human-dominated landscape in Central Veracruz, Mexico. Environmental Entomology, 44(6), 1488-1497.

García-Martínez, M. A., Valenzuela-González, J. E., Escobar-Sarria, F., López-Barrera, F., \& Castaño-Meneses, G. (2017). The surrounding landscape influences the diversity of leaf-litter ants in riparian cloud forest remnants. PLoS ONE, 12(2), 1-19.

Global Forest Watch. (2019). Tree cover loss in Brazil. Recuperado de https:/www.globalforestwatch.org/ dashboards/country/BRA?category=forest-change

Gollan, J. R., De Bruyn, L. L., Reid, N., Smith, D., \& Wilkie, L. (2011). Can ants be used as ecological indicators of restoration progress in dynamic environments? A case study in a revegetated riparian zone. Ecological Indicators, 11(6), 1517-1525.

Hölldobler, B., \& Wilson, E. O. (1994). Journey to the ants: a story of scientific exploration. Cambridge, England: Harvard University Press.

Hothorn, T., Bretz, F., Westfall, P., Heiberger, R. M., Schuetzenmeister, A., \& Scheibe, S. (2016). Package "multcomp" Version 1.4-6. Recuperado de https://cran.r-project.org/web/packages/multcomp/ multcomp.pdf

IBGE - Instituto Brasileiro de Geografia e Estatística. (2004). Biomas. Recuperado de https://www.ibge. gov.br/geociencias/informacoes-ambientais/estudosambientais/15842-biomas.html? $=\& \mathrm{t}=$ o-que-e Último acceso 16/07/2019.

INPE - Instituto Nacional de Pesquisas Espaciais. (2019). Projeto Prodes, monitoramento da floresta amazônica brasileira por satélite. Recuperado de http://www.obt. inpe.br/OBT/assuntos/programas/amazonia/prodes

Jones, C. G., Lawton, J. H., \& Shachak, M. (1994). Organisms as ecosystem engineers. Oikos, 69(3), 373-386.

Leal, C. R. O., Silva, J. O., Sousa-Souto, L., \& Neves, F. de S. (2016). Vegetation structure determines insect herbivore diversity in seasonally dry tropical forests. Journal of Insect Conservation, 20(6), 979-988.
Leal, I. R., Leal, L. C., \& Andersen, A. N. (2015). The benefits of myrmecochory: A matter of stature. Biotropica, 47(3), 281-285.

Legendre, P., Borcard, D., \& Peres-Neto, P. R. (2005). Analyzing beta diversity: partitioning the spatial variation of community composition data. Ecological Monographs, 75(4), 435-450.

Lima, W. P., \& Zakia, M. J. B. (2004). Hidrologia de Matas Ciliares. In R. R. Rodrigues, \& H. LeitãoFilho, Matas Ciliares: Conservação e Recuperação (pp. 33-44). São Paulo, Brasil: Universidade de São Paulo.

Longino, J. T., Coddington, J., \& Colwell, R. K. (2002). The ant fauna of a tropical rain forest: Estimating species richness three different ways. Ecology, 83(3), 689-702.

Lopes, J. F. S., Hallack, N. M. D. R., Sales, T. A. de, Brugger, M. S., Ribeiro, L. F., Hastenreiter, I. N., \& Camargo, R. D. S. (2012). Comparison of the ant assemblages in three phytophysionomies: Rocky field, secondary forest, and riparian forest - A case study in the state park of Ibitipoca, Brazil. Psyche, (928371), 1-7.

Lutinski, J. A., Lutinski, C. J., Iop, S., \& Garcia, F. R. M. (2013). Evaluation of an ant sampling protocol (Hymenoptera: Formicidae) in three modified environments located inside an austral Atlantic Forest area of Brazil. Ecologia Austral, 37, 37-43.

Melo, A. S. (2004). A critique of the use of jackknife and related non-parametric techniques to estimate species richness. Community Ecology, 5, 149-157.

Minitab Statistical Software. (2018). Minitab 18 Trial version. Recuperado de https://www.minitab.com

Neves, F. S., Queiroz-Dantas, K. S., Rocha, W. D., \& Delabie, J. H. C. D. (2013). Ants of three adjacent habitats of a transition region between the Cerrado and Caatinga biomes: the effects of heterogeneity and variation in canopy cover. Neotropical Entomology, 42(3), 258-268.

Nunes, S. S., Barlow, J., Gardner, T. A., Siqueira, J. V., Sales, M. R., \& Souza, C. M. (2014). A 22 year assessment of deforestation and restoration in riparian forests in the eastern Brazilian Amazon. Environmental Conservation, 42(3), 193-203.

Oksanen, J., Blanchet, F. G., Kindt, R., Legendre, P., Minchin, P. R., ... Oksanen, M. J. (2018). Vegan: community ecology package. R Package (Version 2, 4-6). Recuperado de https://cran.r-project.org/web/ packages/vegan/vegan.pdf

Oliveira, J. A. V., Silva, D. M., \& Santana, F. A. (2014). Ant species diversity in ciliary forest and gallery forest areas in central Brazil. Advances in Entomology, 2(1), 24-32 
Pinzón, S. T., Rousseau, G. X., Rocha, A. P., Celentano, D., Corrêa, M. L. Z., \& Braun, H. (2015). La macrofauna del suelo como indicadora de degradación de bosques ribereños en la amazonia oriental brasilera. Revista de la Facultad de Agronomía - La Plata, 114(1), 49-60.

R Development Core Team. (2009). R: A language and environment for statistical computing. R Foundation for Statistical Computing, Vienna, Austria. Recuperado de http://www.R-project.org

Rodrigues, R. R., Brancalion, P. H. S., \& Isernhagen, I. (2009). Pacto pela Restauração da Mata Atlântica: referencial dos conceitos e ações de restauração florestal. São Paulo, Brasil: LERF/ESALQ - Instituto BioAtlântica.

Rousseau, G. X., Silva, P. R. dos S., Celentano, D., \& Carvalho, C. J. R. de. (2014). Macrofauna do solo em uma cronosequência de capoeiras, florestas e pastos no Centro de Endemismo Belém, Amazônia Oriental Acta Amazonica, 44(4), 499-512.

Schmidt, F. A., \& Solar, R. R. C. (2010). Hypogaeic pitfall traps: methodological advances and remarks to improve the sampling of a hidden ant fauna. Insectes Sociaux, 57(3), 261-266.

Tanaka, K., Ogata, K., Mukai, H., Yamawo, A., \& Tokuda, M. (2015). Adaptive advantage of myrmecochory in the ant-dispersed herb Lamium amplexicaule (Lamiaceae): Predation avoidance through the deterrence of post-dispersal seed predators. PLoS ONE, 10(7), $1-12$.

Vasconcellos, R. L. F., Segat, J. C., Bonfim, J. A., Baretta, D., \& Cardoso, E. J. B. N. (2013). Soil macrofauna as an indicator of soil quality in an undisturbed riparian forest and recovering sites of different ages. European Journal of Soil Biology, 58, 105-112.

Vasconcelos, H. L. (1999). Effects of forest disturbance on the structure of ground-foraging ant communities in Central Amazonia. Biodiversity Conservation, 8, 409-420.

Vasconcelos, H. L., \& Vilhena, J. M. S. (2006). Species turnover and vertical partitioning of ant assemblages in the Brazilian Amazon: a comparison of forests and savannas. Biotropica, 38(1), 100-106.

Vasconcelos, H. L., Vilhena, J. M. S., Magnusson, W. E., \& Albernaz, A. L. K. M. (2006). Long-term effects of forest fragmentation on Amazonian ant communities. Journal of Biogeography, 33(8), 1348-1356.

Vasconcelos, H. L., Frizzo, T. L. M., Pacheco, R., Maravalhas, J. B., Camacho, G. P., Carvalho, K. S., ... PujolLuz, J. R. (2014). Evaluating sampling sufficiency and the use of surrogates for assessing ant diversity in a Neotropical biodiversity hotspot. Ecological Indicators, 46, 286-292.

Whittaker, R. H. (1972). Evolution and measurement of species diversity. Taxon, 21, 213-251.

Wilkie, K. T. R., Mertl, A. L., \& Traniello, J. F. A. (2007). Biodiversity below ground: probing the subterranean ant fauna of Amazonia. Naturwissenschaften, 94(9), 725-731.

Zelarayán, M. L. C., Celentano, D., Oliveira, E. C., Triana, S. P., Sodré, D. N., Muchavisoy, K. H. M., \& Rousseau, G. X. (2015). Impacto da degradação sobre o estoque total de carbono de florestas ripárias na Amazônia Oriental, Brasil. Acta Amazonica, 45(3), 271-282. 